Theoretical and Mathematical Physics, 159(2): 587-597 (2009)

\title{
SPECTRAL PARAMETERIZATION FOR POWER SUMS OF A QUANTUM SUPERMATRIX
}

\author{
(c) D. I. Gurevich, ${ }^{* \dagger}$ P. N. Pyatov, ${ }^{* \dagger}$ and P. A. Saponov ${ }^{\S}$
}

We obtain a parameterization for power sums of a $G L(m \mid n)$-type quantum (super)matrix in terms of its spectral values.

Keywords: quantum (super)matrix, characteristic subalgebra, spectral value

\section{Introduction}

This paper adds to our previous works [1]-[3] devoted to quantum matrix algebras (QMAs) of the $G L(m \mid n)$ type. Here we continue to investigate the commutative characteristic subalgebra of the QMA. More precisely, we find a parameterization for the set of "quantum" traces of "powers" of a quantum (super)matrix in terms of spectral values, which are quantum analogues of the set of (super)matrix eigenvalues. We note that this set of quantum traces generates the characteristic subalgebra. To illustrate our statements, we briefly recall the corresponding facts from the classical matrix algebra.

As is well known, any complex $N \times N$ matrix $M \in \operatorname{Mat}_{N}(\mathbb{C})$ satisfies the polynomial Cayley-Hamilton (or characteristic) identity, which can be represented in the factored form

$$
\prod_{i=1}^{N}\left(M-\mu_{i} I\right)=0,
$$

where $I$ is the unit matrix and $\mu_{i}, i=1, \ldots, N$, are the eigenvalues of $M$. Opening the brackets, we can rewrite this identity as

$$
\sum_{k=0}^{N}(-1)^{k} e_{k}(\mu) M^{N-k}=0,
$$

where $e_{k}(\mu), k=0,1, \ldots, N$, are elementary symmetric polynomials ${ }^{1}$ in the variables $\left\{\mu_{i}\right\}_{1 \leq i \leq N}$. The elementary symmetric polynomials generate the whole algebra of symmetric polynomials in the eigenvalues $\mu_{i}$. Another well-known generating set for symmetric polynomials is given by the power sums

$$
p_{k}(\mu):=\sum_{i=1}^{N} \mu_{i}^{k} \equiv \operatorname{Tr} M^{k} .
$$

\footnotetext{
*Max-Planck-Institut für Mathematik, Bonn, Germany.

${ }^{\dagger}$ Université de Valenciennes, Valenciennes, France, e-mail: gurevich@univ-valenciennes.fr.

${ }^{\ddagger}$ Joint Institute for Nuclear Research, Dubna, Moscow Oblast, Russia, e-mail: pyatov@theor.jinr.ru.

§Institute for High Energy Physics, Protvino, Moscow Oblast, Russia, e-mail: Pavel.Saponov@ihep.ru.

${ }^{1}$ See [4] for a review of basic results on symmetric functions.
}

Prepared from an English manuscript submitted by the authors; for the Russian version, see Teoreticheskaya $i$ Matematicheskaya Fizika, Vol. 159, No. 2, pp. 207-219, May, 2009. 
A relation between the two sets of generators is provided by Newton's recursion

$$
k e_{k}+\sum_{r=1}^{k}(-1)^{r} p_{r} e_{k-r}=0, \quad k \geq 1 .
$$

In [1], [2], we found analogues of the above classical results for a family of Hecke-type QMAs, which includes $q$-generalizations of $G L(m \mid n)$-type supermatrices for all integers $m \geq 0$ and $n \geq 0$ (see the definitions in the next section). In particular, we derived the Cayley-Hamilton identities for these algebras, which clarified the way to introduce the spectral values for quantum matrices. We note that the coefficients of the Cayley-Hamilton polynomials mutually commute. They generate a commutative characteristic subalgebra in the QMA, which is an analogue of the algebra of symmetric polynomials in the eigenvalues of the matrix $M$. The Cayley-Hamilton identity allows representing the elements of the characteristic subalgebra as (super)symmetric polynomials in the spectral values of the quantum matrix.

As in the classical case, quantum analogues of the power sums can be defined as some specific traces of "powers" of the quantum matrix. By construction, the power sums belong to the characteristic subalgebra, but an explicit expression for them in terms of the spectral values was not yet known. Our main goal here is to derive such an expression.

Our presentation is strongly based on the previous works cited above. In the next section, we give the notation and list the definitions and main results used in what follows (see [1], [2] for a more detailed exposition, proofs, and a short overview).

\section{Some basic results and definitions}

Let $V$ be a finite-dimensional linear space over the field of complex numbers $\mathbb{C}, \operatorname{dim} V=N$. Let $I$ denote the identity matrix (its dimension is clear from the context if not explicitly specified) and $P \in \operatorname{Aut}\left(V^{\otimes 2}\right)$ be the permutation automorphism $P(u \otimes v)=v \otimes u$.

With any element $X \in \operatorname{End}\left(V^{\otimes p}\right), p=1,2, \ldots$, we associate a sequence of endomorphisms $X_{i} \in$ $\operatorname{End}\left(V^{\otimes k}\right), k \geq p, i=1, \ldots, k-p+1$, according to the rule

$$
X_{i}=I^{\otimes(i-1)} \otimes X \otimes I^{\otimes^{(k-p-i+1)}}, \quad 1 \leq i \leq k-p+1,
$$

where $I$ is the identity automorphism of $V$.

We consider a pair of invertible operators $R, F \in \operatorname{Aut}\left(V^{\otimes 2}\right)$ subject to the following conditions:

1. The operators $R$ and $F$ satisfy the Yang-Baxter equations

$$
R_{1} R_{2} R_{1}=R_{2} R_{1} R_{2}, \quad F_{1} F_{2} F_{1}=F_{2} F_{1} F_{2} .
$$

Such operators are called $R$-matrices.

2. The pair of $R$-matrices $\{R, F\}$ is compatible, i.e.,

$$
R_{1} F_{2} F_{1}=F_{2} F_{1} R_{2}, \quad F_{1} F_{2} R_{1}=R_{2} F_{1} F_{2}
$$

3. The matrices of both the operators $R$ and $F$ are strictly skew invertible. With the operator $R$ as an example, this requirement means that 
a. $R$ is skew invertible if there exists an operator $\Psi^{R} \in \operatorname{End}\left(V^{\otimes 2}\right)$ such that

$$
\operatorname{Tr}_{(2)} R_{12} \Psi_{23}^{R}=P_{13},
$$

where the subscript on the trace symbol indicates the number of the space $V$ where the trace is evaluated (the component spaces in the tensor product are enumerated as $V^{\otimes k}:=V_{1} \otimes V_{2} \otimes$ $\left.\cdots \otimes V_{k}\right)$, and

b. the strictness condition additionally implies that the operator $D_{1}^{R}:=\operatorname{Tr}_{(2)} \Psi_{12}^{R}$ is invertible.

With the matrix $D^{R}$, we define the $R$-trace operation ${ }^{2}$ as $\operatorname{Tr}_{R}: \operatorname{Mat}_{N}(W) \rightarrow W$,

$$
\operatorname{Tr}_{R} X:=\sum_{i, j=1}^{N} D^{R_{i}^{j}} X_{j}^{i}, \quad X \in \operatorname{Mat}_{N}(W),
$$

where $W$ is an arbitrary linear space.

For a compatible pair $\{R, F\}$ of strictly skew-invertible $R$-matrices, the $Q M A \mathcal{M}(R, F)$ is a unital associative algebra generated by $N^{2}$ components of the matrix $\left\|M_{j}^{i}\right\|_{i=1}^{N}$ satisfying the relations

$$
R_{1} M_{\overline{1}} M_{\overline{2}}=M_{\overline{1}} M_{\overline{2}} R_{1},
$$

where we introduce the notation

$$
M_{\overline{1}}:=M_{1}, \quad M_{\overline{k+1}}:=F_{k} M_{\bar{k}} F_{k}^{-1}
$$

for the copies $M_{\bar{k}}$ of the matrix $M$. Definition (2.3) then implies relations of the same type for consecutive pairs of the copies of $M$ (see [5])

$$
R_{k} M_{\bar{k}} M_{\overline{k+1}}=M_{\bar{k}} M_{\overline{k+1}} R_{k} .
$$

Specific subfamilies in a variety of QMAs are extracted by imposing additional conditions on the $R$-matrix $R$ in definition (2.3). We now describe these conditions.

We suppose that $R$ has a quadratic minimal polynomial that can be suitably normalized ${ }^{3}$ as

$$
(R-q I)\left(R+q^{-1} I\right)=0, \quad q \in \mathbb{C} \backslash 0 .
$$

In this context, this relation is called the Hecke condition, and the $R$-matrices satisfying it are called the Hecke $R$-matrices. We further assume that the parameter $q$ in (2.4) is generic, i.e., it does not coincide with the roots of the equation

$$
k_{q}:=\frac{q^{k}-q^{-k}}{q-q^{-1}}=0
$$

for any $k=2,3, \ldots$

Given any Hecke $R$-matrix $R$, we can construct a series of $R$-matrix representations $\rho_{R}$ of the A-type Hecke algebras $\mathcal{H}_{k}(q) \stackrel{\rho_{R}}{\longrightarrow} \operatorname{End}\left(V^{\otimes k}\right), k=2,3, \ldots$ The characteristic properties of these representations are used to classify the Hecke $R$-matrices. ${ }^{4}$ Without going into the construction details, we only mention that under conditions (2.5), the Hecke algebra $\mathcal{H}_{k}(q)$ is isomorphic to the group algebra $\mathbb{C}\left[S_{k}\right]$ of the symmetric group, that its irreducible representations are labeled by a set of partitions $\lambda \vdash k$, and that the corresponding central idempotents in $\mathcal{H}_{k}(q)$ are denoted by $e^{\lambda}$ in what follows. We fix some decomposition of $e^{\lambda}$ into a sum of primitive idempotents $e_{a}^{\lambda} \in \mathcal{H}_{k}(q): e^{\lambda}=\sum_{a=1}^{d_{\lambda}} e_{a}^{\lambda}$, where $d_{\lambda}$ is the dimension of the representation with the label $\lambda$. It is also suitable to introduce the following notation:

\footnotetext{
${ }^{2}$ In the literature on quantum groups, the $R$-trace is usually called the quantum trace or, briefly, the $q$-trace. Giving a different name to this operation, we hope to avoid a misleading association with the parameter $q$ of the Hecke algebra (see below).

${ }^{3}$ We note that conditions $(2.1)$ and $(2.2)$ are independent of the normalization of $R$.

${ }^{4} \mathrm{~A}$ brief description of the Hecke algebras and their $R$-matrix representations can be found in [1]. See [6], [7] and the references therein for a more detailed exposition of the subject.
} 
For two arbitrary integers $m \geq 0$ and $n \geq 0$, an infinite set of partitions $\lambda=\left(\lambda_{1}, \lambda_{2}, \ldots\right)$ satisfying the restriction $\lambda_{m+1} \leq n$ is denoted by $\mathrm{H}(m, n)$.

The partition $\left((n+1)^{m+1}\right) \vdash(m+1)(n+1)$ is briefly denoted by $\lambda_{m, n}$. The corresponding Young diagram is a rectangle with $m+1$ rows of length $n+1$. We note that $\lambda_{m, n}$ is a minimal partition not belonging to the set $\mathrm{H}(m, n)$.

We are now ready to formulate the classification of the Hecke $R$-matrices.

Proposition 1 [8], [3]. For a generic value of $q$, the set of Hecke $R$-matrices is partitioned into subsets labeled by an ordered pair of nonnegative integers $\{m, n\}$. The $R$-matrices belonging to the subset with the label $\{m, n\}$ are called $G L(m \mid n)$-type matrices (or, in other words, they have the bi-rank $(m \mid n)$ ). The $R$ matrix representations $\rho_{R}$ generated by a $G L(m \mid n)$-type $R$-matrix $R$ have the property that for all integers $k \geq 2$ and for any partition $\nu \vdash k$, the images of the idempotents $e^{\nu} \in \mathcal{H}_{k}(q)$ satisfy the relations

$$
\rho_{R}\left(e^{\nu}\right)=0 \quad \text { iff } \quad \nu \notin \mathrm{H}(m, n)
$$

or, equivalently, iff $\lambda_{m, n} \subset \nu$, where the inclusion $\mu=\left(\mu_{1}, \mu_{2}, \ldots\right) \subset \nu=\left(\nu_{1}, \nu_{2}, \ldots\right)$ means that $\mu_{i} \leq \nu_{i}$ for all $i$.

An algebra $\mathcal{M}(R, F)$ defined by a Hecke $(G L(m \mid n)$-type) $R$-matrix $R$ is called a Hecke $(G L(m \mid n)$-type) QMA.

For the Hecke QMA $\mathcal{M}(R, F)$, we consider a set of its elements $s_{\lambda}(M)$ called the Schur functions,

$$
s_{0}(M):=1, \quad s_{\lambda}(M):=\operatorname{Tr}_{R^{(1 \ldots k)}}\left(M_{\overline{1}} \cdots M_{\bar{p}} \rho_{R}\left(e_{a}^{\lambda}\right)\right), \quad \lambda \vdash p, \quad p=1,2, \ldots,
$$

where the definition of $s_{\lambda}(M)$ is independent of the particular choice of the primitive idempotent $e_{a}^{\lambda}$ (indeed, it can be replaced with $\left.d_{\lambda}^{-1} e^{\lambda}\right)$. As shown in [9], the linear span of the Schur functions $s_{\lambda}(M)$ is an Abelian subalgebra in $\mathcal{M}(R, F)$. In what follows, we call it the characteristic subalgebra of $\mathcal{M}(R, F)$. It follows that the characteristic subalgebra of the $G L(m \mid n)$-type QMA is spanned by the Schur functions $s_{\lambda}(M)$, $\lambda \in \mathrm{H}(m, n)$. The multiplication table for the elements $s_{\lambda}(M) \in \mathcal{M}(R, F)$ coincides with that for the basis of Schur functions in the ring of symmetric functions (see [4]), thus justifying the notation. We have [2]

$$
s_{\lambda}(M) s_{\mu}(M)=\sum_{\nu} C_{\lambda \mu}^{\nu} s_{\nu}(M)
$$

where $C_{\lambda \mu}^{\nu}$ are the Littlewood-Richardson coefficients. Later, we need information about the generating sets of the characteristic subalgebra.

Proposition 2 [9], [5]. For generic values of $q$, the characteristic subalgebra of the Hecke QMA $\mathcal{M}(R, F)$ is generated by any one of the three sets

1. the single-column Schur functions $a_{k}(M):=s_{\left(1^{k}\right)}(M), k=0,1,2, \ldots$,

2. the single-row Schur functions $s_{k}(M):=s_{(k)}(M), k=0,1,2, \ldots$, and

3. the set of power sums

$$
p_{0}(M):=\left(\operatorname{Tr}_{R} I\right) 1, \quad p_{k}(M):=\operatorname{Tr}_{R^{(1 \ldots k)}}\left(M_{\overline{1}} \cdots M_{\bar{k}} R_{k-1} \cdots R_{1}\right), \quad k \geq 1 .
$$


These sets are connected by a series of Newton and Wronski recurrence relations for $k \geq 1$,

$$
\begin{aligned}
& (-1)^{k} k_{q} a_{k}(M)+\sum_{r=0}^{k-1}(-q)^{r} a_{r}(M) p_{k-r}(M)=0, \\
& k_{q} s_{k}(M)-\sum_{r=0}^{k-1} q^{-r} s_{r}(M) p_{k-r}(M)=0, \\
& \sum_{r=0}^{k}(-1)^{r} a_{r}(M) s_{k-r}(M)=0 .
\end{aligned}
$$

Introducing the generating functions for these sets of generators

$$
\begin{aligned}
& A(t):=\sum_{k \geq 0} a_{k}(M) t^{k}, \quad S(t):=\sum_{k \geq 0} s_{k}(M) t^{k}, \\
& P(t):=1+\left(q-q^{-1}\right) \sum_{k \geq 1} p_{k}(M) t^{k},
\end{aligned}
$$

we can rewrite relations (2.8)-(2.10) in the compact form [4], [10 $]^{5}$

$$
P(-t) A(q t)=A\left(q^{-1} t\right), \quad P(t) S\left(q^{-1} t\right)=S(q t), \quad A(t) S(-t)=1 .
$$

For the $G L(m \mid n)$-type QMA, the zeroth-power sum is [3]

$$
p_{0}(M)=q^{n-m}(m-n)_{q} 1 .
$$

One of the remarkable properties of the Hecke QMA $\mathcal{M}(R, F)$ is the existence of the characteristic identity for the matrix $M$ of its generators. To formulate the result, we introduce the notion of the matrix $\star$-product of quantum matrices (see Sec. 4.4 in [12] for a detailed exposition). Namely, starting with the quantum matrix of generators $M$ and the scalar quantum matrices $s_{\lambda}(M) I$ for all $\lambda \vdash k, k \geq 0$, we construct the whole set of quantum matrices by the following recursive procedure: given any quantum matrix $N$, its $\star$-multiplication by $s_{\lambda}(M) I$ and left $\star$-multiplication by $M$ are also quantum matrices defined as

$$
\begin{aligned}
& M \star\left(s_{\lambda}(M) I\right)=\left(s_{\lambda}(M) I\right) \star M:=M \cdot s_{\lambda}(M), \\
& M \star N:=M \cdot \phi(N), \quad \text { where } \phi(N)_{1}:=\operatorname{Tr}_{R^{(2)}} N_{\overline{2}} R_{12}
\end{aligned}
$$

and the dot product is the usual multiplication of a matrix by a scalar (matrix). The $\star$-product of quantum matrices is required to be associative; it is commutative by construction. The $\star$-powers of a quantum matrix $M$ are

$$
\begin{aligned}
& M^{\overline{0}}:=I, \quad M^{\overline{1}}:=M, \\
& M^{\bar{k}}:=\underbrace{M \star \cdots \star M}_{k \text { matrices }}=\operatorname{Tr}_{R^{(2 \ldots k)}\left(M_{\overline{1}} \cdots M_{\bar{k}} R_{k-1} \cdots R_{1}\right), \quad k>1 .}^{M},
\end{aligned}
$$

We note that for the family of the so-called reflection equation algebras, the QMAs of the form $\mathcal{M}(R, R)$, the $\star$-product coincides with the usual matrix product.

The characteristic identity depends essentially on the type of the QMA. For the $G L(m \mid n)$-type QMA, it is an $(m+n)$ th-order polynomial identity in $\star$-powers of the matrix $M$ with coefficients in the characteristic subalgebra. The following $q$-analogue of the classical Cayley-Hamilton theorem holds.

\footnotetext{
${ }^{5}$ The first of these relations was also derived in the limit case $\mathcal{M}(R, R) \stackrel{q \rightarrow 1}{\longrightarrow} U\left(\mathfrak{g l}_{n}\right)$ in [11].
} 
Theorem 1 [1], [2]. The characteristic identity for the matrix of generators of the $G L(m \mid n)$-type $Q M A \mathcal{M}(R, F)$ is given by

$$
\left(\sum_{k=0}^{m}(-q)^{k} M^{\overline{m-k}} s_{[m \mid n]^{k}}(M)\right) \star\left(\sum_{r=0}^{n} q^{-r} M^{\overline{n-r}} s_{[m \mid n]_{r}}(M)\right) \equiv 0,
$$

where we use the brief notation for the partitions

$$
[m \mid n]^{k}:=\left((n+1)^{k}, n^{m-k}\right), \quad[m \mid n]_{r}:=\left(n^{m}, r\right) .
$$

Remarkably, for a generic-type QMA (i.e., if $m n>0$ ), the characteristic polynomial in the left-hand side of identity (2.14) factors into the product of two factors of degrees $m$ and $n$. Therefore, in setting the factorization problem for the characteristic polynomial, we must separate all the $m+n$ roots into two parts of sizes $m$ and $n$.

Let $\mathbb{C}[\mu, \nu]$ be the algebra of polynomials in two sets of mutually commuting and algebraically independent variables $\mu:=\left\{\mu_{i}\right\}_{1 \leq i \leq m}$ and $\nu:=\left\{\nu_{j}\right\}_{1 \leq j \leq n}$. We consider the map of the coefficients of the characteristic polynomial into $\mathbb{C}[\mu, \nu]$

$$
\begin{array}{ll}
s_{[m \mid n]^{k}}(M) \mapsto s_{[m \mid n]^{k}}(\mu, \nu):=s_{[m \mid n]}(\mu, \nu) e_{k}\left(q^{-1} \mu\right), & 1 \leq k \leq m, \\
s_{[m \mid n]_{r}}(M) \mapsto s_{[m \mid n]_{r}}(\mu, \nu):=s_{[m \mid n]}(\mu, \nu) e_{r}(-q \nu), & 1 \leq r \leq n,
\end{array}
$$

where $e_{k}(\cdot)$ are elementary symmetric polynomials in their arguments (e.g., $e_{k}(\mu) \equiv e_{k}\left(\mu_{1}, \ldots, \mu_{m}\right)=$ $\left.\sum_{1 \leq i_{1}<\cdots<i_{k} \leq m} \mu_{i_{1}} \cdots \mu_{i_{k}}\right)$. For the moment, we do not specify an explicit expression for the polynomial $s_{[m \mid n]}(\mu, \nu)$. We now define a central extension of the $\star$-product algebra of the quantum matrices by scalar matrices of the form $p(\mu, \nu) I, p(\mu, \nu) \in \mathbb{C}[\mu, \nu]$, such that $s_{\lambda}(M) I \equiv s_{\lambda}(\mu, \nu) I$. In the extended algebra, characteristic identity (2.14) has the completely factored form

$$
\prod_{i=1}^{m}\left(M-\mu_{i} I\right) \star \prod_{j=1}^{n}\left(M-\nu_{j} I\right) \cdot\left(s_{[m \mid n]}(\mu, \nu)\right)^{2} \equiv 0 .
$$

Assuming that $s_{[m \mid n]}(\mu, \nu) \neq 0$, we can interpret the variables $\mu_{i}, i=1, \ldots, m$, and $\nu_{j}, j=1, \ldots, n$, as eigenvalues of the quantum matrix $M$, respectively called the even and odd spectral values of $M$.

Map (2.15), (2.16) admits a unique extension to a homomorphic map of the characteristic subalgebra into the algebra $\mathbb{C}[\mu, \nu]$ of polynomials in the spectral values $\mu_{i}$ and $\nu_{j}$. Using Littlewood-Richardson multiplication rule (2.6), we obtain (see [2])

$$
\begin{array}{ll}
a_{k}(M) \equiv s_{[k \mid 1]}(M) & \mapsto \quad a_{k}(\mu, \nu):=\sum_{r=0}^{k} e_{r}\left(q^{-1} \mu\right) h_{k-r}(-q \nu), \\
s_{k}(M) \equiv s_{[1 \mid k]}(M) & \mapsto \quad s_{k}(\mu, \nu):=\sum_{r=0}^{k} e_{r}(-q \nu) h_{k-r}\left(q^{-1} \mu\right),
\end{array}
$$

where $h_{k}(\cdot)$ denotes the complete symmetric polynomial in its variables, $h_{k}(\mu) \equiv h_{k}\left(\mu_{1}, \ldots, \mu_{m}\right)=$ $\sum_{1 \leq i_{1} \leq \cdots \leq i_{k} \leq m} \mu_{i_{1}} \cdots \mu_{i_{k}}$.

Because each of the sets $\left\{a_{k}(M)\right\}_{k \geq 0}$ and $\left\{s_{k}(M)\right\}_{k \geq 0}$ generates the characteristic subalgebra, the homomorphism is completely defined by (2.17) or by (2.18). In particular, formulas (2.17) and (2.18) imply 
an explicit expression for the unspecified polynomial $s_{[m \mid n]}(\mu, \nu)$ in $(2.15)$ and (2.16), which is the image of $s_{[m \mid n]}(M)$ :

$$
s_{[m \mid n]}(\mu, \nu)=\prod_{i=1}^{m} \prod_{j=1}^{n}\left(q^{-1} \mu_{i}-q \nu_{j}\right) .
$$

This homomorphic map induced by (2.17) or (2.18) is called the spectral parameterization of the characteristic subalgebra. In the next section, we derive the spectral parameterization for the third generating set of the characteristic subalgebra, i.e., the set of power sums $\left\{p_{k}(M)\right\}_{k \geq 0}$.

\section{Spectral parameterization of power sums}

In this section, we work with the $G L(m \mid n)$-type QMA $\mathcal{M}(R, F)$ defined by relations (2.3) with an $R$ matrix $R$ satisfying the criterion in Proposition 1. During our consideration, we assume that the parameter $q$ is generic (see (2.5)), although this restriction can subsequently be waived: unlike the Schur functions $a_{k}(M)$ and $s_{k}(M)$, the power sums $p_{k}(M)$ are consistently defined for all $q \in \mathbb{C} \backslash 0$.

The spectral parameterization of the power sums was found in [13] in the particular case of the $G L(m):=G L(m \mid 0)$-type reflection equation algebra $\mathcal{M}(R, R)$. Taking into account that the quantum matrix has only "even" eigenvalues $\left\{\mu_{i}\right\}_{1 \leq i \leq m}$ in the $G L(m)$ case, we write the result as

$$
p_{k}(M) \mapsto \sum_{i=1}^{m} d_{i} \mu_{i}^{k}, \quad \text { where } d_{i}:=q^{-1} \prod_{j \neq i}^{m} \frac{\mu_{i}-q^{-2} \mu_{j}}{\mu_{i}-\mu_{j}} .
$$

Our goal is to extend this formula to the general case. For this, we introduce an auxiliary set of polynomials $\left\{\pi_{k}(\mu, \nu)\right\}_{k \geq 1} \subset \mathbb{C}[\mu, \nu]$ defined by the relations

$$
\pi_{k}(\mu, \nu):=\sum_{i=1}^{m}\left(q^{-1} \mu_{i}\right)^{k}-\sum_{j=1}^{m}\left(q \nu_{j}\right)^{k}, \quad k \geq 1 .
$$

In what follows, we use the following property of these polynomials.

Lemma 1. The sets of polynomials $\left\{a_{k}(\mu, \nu)\right\}_{k \geq 0},\left\{s_{k}(\mu, \nu)\right\}_{k \geq 0}$ (see Eqs. (2.17) and (2.18)), and $\left\{\pi_{k}(\mu, \nu)\right\}_{k \geq 1}$ satisfy the Newton recurrence relations

$$
\begin{aligned}
& (-1)^{k} k a_{k}(\mu, \nu)+\sum_{r=0}^{k-1}(-1)^{r} a_{r}(\mu, \nu) \pi_{k-r}(\mu, \nu)=0, \\
& k s_{k}(\mu, \nu)-\sum_{r=0}^{k-1} s_{r}(\mu, \nu) \pi_{k-r}(\mu, \nu)=0, \quad k \geq 1 .
\end{aligned}
$$

Proof. First, we recall the relations between the generating functions for the power sums and the elementary symmetric and complete symmetric polynomials in a finite set of variables $x:=\left\{x_{i}\right\}_{1 \leq i \leq p}$ (see Sec. I.2 in [4])

$$
\begin{aligned}
E(x \mid t) & :=\sum_{k=0}^{p} e_{k}(x) t^{k}=\prod_{i=1}^{p}\left(1+x_{i} t\right), \\
H(x \mid t) & :=\sum_{k \geq 0} h_{k}(x) t^{k}=\prod_{i=1}^{p}\left(1-x_{i} t\right)^{-1}, \\
P(x \mid t) & :=\sum_{k \geq 1} p_{k}(x) t^{k-1}=-\frac{d}{d t} \log E(x \mid-t)=\frac{d}{d t} \log H(x \mid t) .
\end{aligned}
$$


We consider three functions depending on the two sets of variables $x:=\left\{x_{i}\right\}_{1 \leq i \leq m}$ and $y:=\left\{y_{i}\right\}_{1 \leq i \leq n}$ :

$$
\begin{aligned}
& A(x, y \mid t):=E(x \mid t) H(-y \mid t), \\
& S(x, y \mid t):=H(x \mid t) E(-y \mid t), \\
& \Pi(x, y \mid t):=P(x \mid t)-P(y \mid t) .
\end{aligned}
$$

These functions are supermatrix analogues of the respective generating functions of the elementary and complete symmetric polynomials and the power sums (see Exercise 27 in Sec. I.5 in [4] and the references therein). Indeed, using (3.4), we can easily verify that these functions satisfy relations similar to (3.4),

$$
\Pi(x, y \mid t)=-\frac{d}{d t} \log A(x, y \mid-t)=\frac{d}{d t} \log S(x, y \mid t) .
$$

The assertion in the lemma now follows because $A\left(q^{-1} \mu, q \nu \mid t\right), S\left(q^{-1} \mu, q \nu \mid t\right)$, and $\Pi\left(q^{-1} \mu, q \nu \mid t\right)$ are the respective generating functions for the sets of polynomials $\left\{a_{k}(\mu, \nu)\right\}_{k \geq 0},\left\{s_{k}(\mu, \nu)\right\}_{k \geq 0}$, and $\left\{\pi_{k}(\mu, \nu)\right\}_{k \geq 1}$. Relations (3.2) and (3.3) are just expansions of (3.6) in powers of $t$.

We can now formulate our main result regarding the power sums.

Proposition 3. Spectral parameterization (2.17) (or (2.18)) for the power sums $p_{k}(M)$ in (2.7) in the $G L(m \mid n)$-type $Q M A$ is given by the formulas

$$
p_{k}(M) \mapsto p_{k}(\mu, \nu)=\sum_{i=1}^{m} d_{i} \mu_{i}^{k}+\sum_{j=1}^{n} \tilde{d}_{j} \nu_{j}^{k}, \quad k \geq 0,
$$

where the "weight" coefficients $d_{i}$ and $\tilde{d}_{j}$ have the explicit forms

$$
\begin{aligned}
& d_{i}:=q^{-1} \prod_{\substack{p=1, p \neq i}}^{m} \frac{\mu_{i}-q^{-2} \mu_{p}}{\mu_{i}-\mu_{p}} \prod_{j=1}^{n} \frac{\mu_{i}-q^{2} \nu_{j}}{\mu_{i}-\nu_{j}}, \\
& \tilde{d}_{j}:=-q \prod_{i=1}^{n} \frac{\nu_{j}-q^{-2} \mu_{i}}{\nu_{j}-\mu_{i}} \prod_{\substack{p=1 \\
p \neq j}}^{n} \frac{\nu_{j}-q^{2} \nu_{p}}{\nu_{j}-\nu_{p}} .
\end{aligned}
$$

Because the spectral values $\left\{\mu_{i}\right\}$ and $\left\{\nu_{j}\right\}$ are assumed to be algebraically independent, all the coefficients $d_{i}$ and $\tilde{d}_{j}$ are nonzero and well defined.

Proof. For the proof, we need yet another set of recurrence relations for the power sums $\left\{p_{k}(\mu, \nu)\right\}_{k \geq 1}$ in (3.7) and the polynomials $\left\{\pi_{k}(\mu, \nu)\right\}_{k \geq 1}$ in (3.1).

Lemma 2. The relations

$$
k p_{k}(\mu, \nu)=k_{q} \pi_{k}(\mu, \nu)+\left(q-q^{-1}\right) \sum_{r=1}^{k-1} r_{q} \pi_{r}(\mu, \nu) p_{k-r}(\mu, \nu), \quad k \geq 1,
$$

hold. In terms of the generating functions

$$
P(\mu, \nu \mid t):=1+\left(q-q^{-1}\right) \sum_{k \geq 1} p_{k}(\mu, \nu) t^{k}, \quad \Pi\left(q^{-1} \mu, q \nu \mid t\right):=\sum_{k \geq 1} \pi_{k}(\mu, \nu) t^{k-1},
$$

where $P(\mu, \nu \mid t)$ is the spectral parameterization of $P(t)$ (see (2.11)) and $\Pi(\mu, \nu \mid t)$ is defined in (3.5), relations (3.10) have the brief form

$$
P(\mu, \nu \mid t)\left(q \Pi\left(q^{-1} \mu, q \nu \mid q t\right)-q^{-1} \Pi\left(q^{-1} \mu, q \nu \mid q^{-1} t\right)\right)=\frac{d}{d t} P(\mu, \nu \mid t) .
$$


Proof of the lemma. We introduce the meromorphic function $f: \mathbb{C}[\mu, \nu] \rightarrow \mathbb{C}(\mu, \nu)$ by the formula

$$
f(z):=\prod_{i=1}^{m} \frac{\left(z-q^{-2} \mu_{i}\right)}{\left(z-\mu_{i}\right)} \prod_{j=1}^{n} \frac{\left(z-q^{2} \nu_{j}\right)}{\left(z-\nu_{j}\right)} .
$$

Because the spectral values are algebraically independent, this function has a first-order pole at each point $\mu_{i}$ and $\nu_{j}$. Moreover, as can be easily seen, $f(0)=q^{2(n-m)}$ and $\lim _{z \rightarrow \infty} f(z)=1$. Taking this limit at infinity into account, we expand the function $f(z)$ into the sum of simple fractions

$$
f(z)=1+\left.\sum_{i=1}^{m} \frac{1}{z-\mu_{i}} \operatorname{Res} f(z)\right|_{z=\mu_{i}}+\left.\sum_{j=1}^{n} \frac{1}{z-\nu_{j}} \operatorname{Res} f(z)\right|_{z=\nu_{j}}
$$

where the residues at the poles are

$$
\left.\operatorname{Res} f(z)\right|_{z=\mu_{i}}=\left(q-q^{-1}\right) \mu_{i} d_{i},\left.\quad \operatorname{Res} f(z)\right|_{z=\nu_{j}}=\left(q-q^{-1}\right) \nu_{j} \tilde{d}_{j} .
$$

Evaluating the right-hand side of (3.12) at $z=0$, we obtain

$$
f(0)=q^{2(n-m)}=1-\left(q-q^{-1}\right)\left(\sum_{i=1}^{m} d_{i}+\sum_{j=1}^{n} \tilde{d}_{j}\right):=1-\left(q-q^{-1}\right) p_{0}(\mu, \nu),
$$

and therefore

$$
p_{0}(\mu, \nu)=q^{n-m}(m-n)_{q} 1 .
$$

We have thus verified the consistency of (3.7) with our previous result $(2.13)$ for $p_{0}(M)$.

To prove relations (3.10), we expand the functions $z^{k} f(z), k \geq 1$, into the sum of simple fractions. In addition to the simple poles at $\mu_{i}$ and $\nu_{j}$, the function $z^{k} f(z)$ has the $k$ th order pole at $z=\infty$ or, in terms of the new variable $y=z^{-1}$, at the point $y=0$. Taking into account that

$$
\left.\operatorname{Res} z^{k} f(z)\right|_{z=\mu_{i}}=\left.\left(\mu_{i}\right)^{k} \operatorname{Res} f(z)\right|_{z=\mu_{i}},\left.\quad \operatorname{Res} z^{k} f(z)\right|_{z=\nu_{j}}=\left.\left(\nu_{j}\right)^{k} \operatorname{Res} f(z)\right|_{z=\nu_{j}},
$$

we obtain the corresponding expansion

$$
z^{k} f(z)=\sum_{r=0}^{k} \frac{z^{k-r}}{r !} f_{r}+\left(q-q^{-1}\right)\left(\sum_{i=1}^{m} \frac{d_{i} \mu_{i}^{k+1}}{z-\mu_{i}}+\sum_{j=1}^{n} \frac{\tilde{d}_{i} \nu_{j}^{k+1}}{z-\nu_{i}}\right),
$$

where the coefficients $f_{r}$ are the derivatives

$$
f_{r}:=\left.\frac{d^{r} f(y)}{d y^{r}}\right|_{y=0}
$$

Evaluating (3.13) at $z=0$ and taking (3.7) into account, we obtain the relation

$$
p_{k}(\mu, \nu)=\frac{f_{k}}{\left(q-q^{-1}\right) k !}, \quad k \geq 1 .
$$

We now calculate $f_{k}$. Recalling that $y=z^{-1}$, we write the first-order derivative $f^{\prime}(y)$ in the form

$$
\frac{d f(y)}{d y}=\left(q-q^{-1}\right) u(y) f(y),
$$


where the function $u(y)$ is given by

$$
u(y):=\sum_{i=1}^{m} \frac{q^{-1} \mu_{i}}{\left(1-\mu_{i} y\right)\left(1-q^{-2} \mu_{i} y\right)}-\sum_{j=1}^{n} \frac{q \nu_{j}}{\left(1-\nu_{j} y\right)\left(1-q^{2} \nu_{j} y\right)} .
$$

By simple induction on $k$, we can verify that

$$
\begin{aligned}
u_{k}(y):=\frac{d^{k} u(y)}{d y^{k}}= & k !\left\{\sum_{i=1}^{m} \sum_{a=0}^{k} \frac{q^{-1-2(k-a)} \mu_{i}^{k+1}}{\left(1-\mu_{i} y\right)^{1+a}\left(1-q^{-2} \mu_{i} y\right)^{1+k-a}}-\right. \\
& \left.-\sum_{j=1}^{n} \sum_{a=0}^{k} \frac{q^{1+2(k-a)} \nu_{j}^{k+1}}{\left(1-\nu_{j} y\right)^{1+a}\left(1-q^{2} \nu_{j} y\right)^{1+k-a}}\right\} .
\end{aligned}
$$

This relation leads immediately to

$$
\begin{aligned}
u_{k}(0) & =k !(k+1)_{q}\left\{\sum_{i=1}^{m}\left(q^{-1} \mu_{i}\right)^{k+1}-\sum_{j=1}^{n}\left(q \nu_{j}\right)^{k+1}\right\}:= \\
& :=k !(k+1)_{q} \pi_{k+1}(\mu, \nu), \quad k \geq 0 .
\end{aligned}
$$

Differentiating relation $(3.15)$ at $y=0$ now yields

$$
f_{k}=\left(q-q^{-1}\right) \sum_{r=0}^{k-1}\left(\begin{array}{c}
r \\
k-1
\end{array}\right) u_{r}(0) f_{k-r-1}, \quad k \geq 1,
$$

where we use the obvious condition $f_{0}=1$. Taking relations (3.14) and (3.16) into account, we can easily prove the assertion in the lemma. Indeed,

$$
\begin{aligned}
k p_{k}(\mu, \nu) & =\frac{f_{k}}{\left(q-q^{-1}\right)(k-1) !}=\frac{1}{(k-1) !} \sum_{r=0}^{k-1}\left(\begin{array}{c}
r \\
k-1
\end{array}\right) u_{r}(0) f_{k-r-1}= \\
& =\sum_{r=0}^{k-1} \frac{(r+1)_{q}}{(k-r-1) !} \pi_{r+1}(\mu, \nu) f_{k-r-1}=\sum_{r=1}^{k} r_{q} \pi_{r}(\mu, \nu) \frac{f_{k-r}}{(k-r) !}= \\
& =k_{q} \pi_{k}(\mu, \nu)+\left(q-q^{-1}\right) \sum_{r=1}^{k-1} r_{q} \pi_{r}(\mu, \nu) p_{k-r}(\mu, \nu) .
\end{aligned}
$$

Finally, the equivalence of (3.10) and (3.11) is verified by a direct calculation.

We are now ready to prove the proposition. We verify that relations (2.8) and (2.9) become identities if we replace $a_{r}(M), s_{r}(M)$, and $p_{k-r}(M)$ with their spectral parameterizations (2.17), (2.18), and (3.7)-(3.9) and use equalities (3.2) and (3.3) together with (3.10). For definiteness, we verify relation (2.8).

To simplify the calculation, we work with the generating functions $A\left(q^{-1} \mu, q \nu \mid t\right)$ for $\left\{a_{k}(\mu, \nu)\right\}_{k \geq 0}$, $\Pi\left(q^{-1} \mu, q \nu \mid t\right)$ for $\left\{\pi_{k}(\mu, \nu\}_{k \geq 1}\right.$, and $P(\mu, \nu \mid t)$ for $\left\{p_{k}(\mu, \nu)\right\}_{k \geq 1}$. Substituting the expression for $\Pi(\cdot)$ in terms of $A(\cdot)$ (see (3.6)) in equality (3.11) and combining like terms, we obtain

$$
\frac{d}{d t} \log \left(P(\mu, \nu \mid t) A\left(q^{-1} \mu, q \nu \mid-q t\right)\right)=\frac{d}{d t} \log \left(A\left(q^{-1} \mu, q \nu \mid-q^{-1} t\right)\right) .
$$

Integrating this equation leads to

$$
P(\mu, \nu \mid t) A\left(q^{-1} \mu, q \nu \mid-q t\right)=C A\left(q^{-1} \mu, q \nu \mid-q^{-1} t\right) .
$$

The obvious boundary condition $P(\mu, \nu \mid 0)=A\left(q^{-1} \mu, q \nu \mid 0\right)=1$ fixes the integration constant $C=1$, and we obtain the sought relation: the first relation in (2.12). 
Acknowledgments. Two of the authors (D. I. G. and P. N. P.) acknowledge the warm hospitality of the Max-Planck-Institut für Mathematik, where this work was completed.

This work is supported in part by l'Agence Nationale de la Recherche (Grant No. ANR-05-BLAN0029-01, D. I. G.) and the Russian Foundation for Basic Research (Grant No. 08-01-00392-a, P. N. P. and P. A. S.).

\section{REFERENCES}

1. D. I. Gurevich, P. N. Pyatov, and P. A. Saponov, St. Petersburg Math. J., 17, 119-135 (2006); arXiv:math.QA/ 0412192v2 (2004).

2. D. I. Gurevich, P. N. Pyatov, and P. A. Saponov, Theor. Math. Phys., 147, 460-485 (2006); arXiv:math.QA/ 0508506v2 (2005).

3. D. I. Gurevich, P. N. Pyatov, and P. A. Saponov, St. Petersburg Math. J., 20, 213-253 (2009); arXiv:math.QA/ 0612815v2 (2006).

4. I. G. Macdonald, Symmetric Functions and Hall Polynomials, Clarendon, Oxford (1995).

5. A. P. Isaev, O. V. Ogievetsky, and P. N. Pyatov, J. Phys. A, 32, L115-L121 (1999); arXiv:math.QA/9809170v1 (1998).

6. A. Ram, Proc. London Math. Soc., 75, 99-133 (1997); arXiv:math.RT/9511223v1 (1995).

7. O. Ogievetsky and P. Pyatov, "Lecture on Hecke algebras," in: Symmetries and Integrable Systems (Proc. Intl. School, Dubna, Russia, June 8-11, 1999, S. Z. Pakulyak, ed.), Joint Inst. Nucl. Res., Dubna (2000), pp. 39-88; Preprint MPIM 2001-40, http://www.mpim-bonn.mpg.de/html/preprints/preprints.html (2001).

8. P. H. Hai, Acta Math. Vietnam., 24, 235-246 (1999).

9. A. Isaev, O. Ogievetsky, and P. Pyatov, Czechoslovak J. Phys., 48, 1369-1374 (1998); arXiv:math.QA/ 9809047v1 (1998).

10. A. Isaev and P. Pyatov, Comm. Math. Phys., DOI: 10.1007/s00220-009-0785-5 (2009); arXiv:0812.2225v1 [math.QA] (2008).

11. T. Umeda, Proc. Amer. Math. Soc., 126, 3169-3175 (1998).

12. O. V. Ogievetsky and P. N. Pyatov, "Orthogonal and symplectic quantum matrix algebras and Cayley-Hamilton theorem for them," arXiv:math.QA/0511618v1 (2005).

13. D. Gurevich and P. Saponov, "Geometry of non-commutative orbits related to Hecke symmetries," in: Quantum Groups (Contemp. Math., Vol. 433, P. Etingof, S. Gelaki, and S. Shnider, eds.), Amer. Math. Soc., Providence, R. I. (2007), pp. 209-250; arXiv:math.QA/0411579v1 (2004). 